\title{
Satisfacción con la democracia en Chile: De lo normativo a lo valorativo
}

\author{
[Satisfaction with democracy in Chile: From norms to values] \\ Karina Cereceda-Marambio ${ }^{1} \&$ Amanda Torres-Solís ${ }^{2}$ \\ ${ }^{1}$ Universidad Diego Portales; 2 Universidad de Chile
}

\begin{abstract}
Resumen
En la presente investigación se propone y contrasta empíricamente un modelo teórico para explicar y predecir la satisfacción con la democracia en Chile. Dicho modelo cuenta con siete variables independientes o predictoras, referidas a la percepción de elementos institucionales-formales (i.e., la percepción de la economía personal y familiar, y la confianza en las instituciones), preferencias políticas, valoración presidencial, percepción de la corrupción y aspectos valorativos sobre derechos y libertades. Así, el modelo propuesto considera la influencia de aspectos personales, valorativos y contingentes en la satisfacción con la democracia. Los resultados de esta investigación indican que la satisfacción con la democracia en Chile se sustenta principalmente en el aspecto institucional macro como la confianza en las instituciones y la economía país, por lo que las variables que miden aspectos del orden valórico poseen menos relevancia para la evaluación del régimen político.
\end{abstract}

Palabras clave: satisfacción con la democracia; percepción económica; confianza en las instituciones; preferencia política; derechos y libertades.

\begin{abstract}
In the current paper, a model to explain and predict the satisfaction about democracy in Chile is proposed and tested against data. This model has seven independent or predictive variables, referring to the perception of institutional-formal elements (the perception of personal economy, country economics and confidence in institutions), political preferences, presidential assessment, perception of corruption and values aspects of rights and freedoms. Thus, the model assessed the influence of values, as well as personal and contingent factors on the satisfaction with democracy. The results indicate that the satisfaction with democracy in Chile is based in the institutional macro aspects such as, trust in the institutions and economic performance; whereas value order variables were found to be less relevant for the political regime assessment..
\end{abstract}

Keywords: satisfaction with democracy; perception of economy; personal economy; political preferences; institutions; values.

Financiamiento: Amanda Torres-Solís agradece el financiamiento recibido del Centro de Conflicto y Cohesión Social [COES] del Fondo COES-FONDAP 15130009, para la realización de esta investigación.

Contacto: La comunicación sobre este artículo debe ser enviada a Karina Cereceda-Marambio. Email: karina.cereceda@ug.uchile.cl 


\section{INTRODUCCIÓN}

En América Latina la democracia ha experimentado amenazas y obstáculos que le impiden fortalecerse y consolidarse, debido a que el pasado autoritario ha generado, por ejemplo, instituciones democráticas débiles, sistemas de partidos políticos con poca credibilidad, una clase política con poca capacidad de adaptarse a los cambios que la sociedad requiere. Pese a esto, en los últimos 25 años se ha desarrollado un proceso de consolidación de la democracia en Chile.

Ya que la satisfacción con la democracia es una de las principales categorías que influyen en la legitimación de la democracia como sistema político (Sanhueza, Cea, \& Guerrero, 2015), es necesario comprender, analizar y evaluar los factores que la explican como una forma de comprender el proceso de consolidación democrática del país.

De este modo, el presente estudio busca dar cuenta de los principales factores que explican la satisfacción con la democracia en Chile, entendiendo que ésta no solo refleja el funcionamiento de la democracia y sus instituciones, sino también muestra dimensiones valóricas y personales relevantes para comprender la evaluación que los sujetos hacen del sistema político.

Calidad de la democracia: De lo global a lo local

Después de la tercera ola de democratización (Huntington, 1994) se han formado numerosas nuevas democracias en el mundo, sin embargo, estas varían enormemente en cuanto a sus características y formas de consolidación, por lo que se ha centrado el debate en la calidad de la democracia (Altman \& Pérez-Liñán, 2002).

Desde la sociología y la ciencia política se ha teorizado sobre el concepto de democracia y sus implicancias como régimen político para la vida en sociedad. Frente a esto, se han descrito tres grandes enfoques, el primero, denominado enfoque procedimental, busca evaluar los procesos e instituciones que sirven para escoger a los gobernantes mediante el sufragio (Tusell, 2015). La propuesta se basa en las reglas del juego bajo la cual los aspirantes al poder compiten entre ellos, asegurando que el acceso a cargos políticos sea lo más limpio y transparente posible (Altman \& Pérez-Liñán, 2002; Mainwaring \& Pérez-Liñán, 2008).

Un segundo enfoque añade al anterior la noción de instituciones de control del poder político para lo cual autores como O'Donnell (2000) incluyen al Estado de derecho como factor de control proponiendo límites y un marco jurídico que garantice los derechos y libertades, agregando que para el buen funcionamiento democrático es necesario que el poder se ejerza sujeto a control. Asimismo, plantea al Estado moderno como un ordenamiento administrativo y jurídico, que permite la regulación a través de una legislación sujeta a las instituciones y normas que lo componen. Cabe señalar que el control del poder se materializa, por una parte, en el accountability -rendición de cuentas- y por otra parte en la supervisión de instituciones 
Cereceda-Marambio, K., \& Torres-Solís, A. (2017). Satisfacción con la democracia en Chile: De lo normativo a lo valorativo. Revista de Sociología 32(1), 32-49. doi: 10.5354/0719-529x.2017.47884

estatales con la capacidad de omitir o sancionar actos (O’Donnell, 2000).

Por último, un tercer enfoque amplía aún más la perspectiva agregando a esta el cumplimiento de objetivos y resultados de la democracia, como por ejemplo, la igualdad socioeconómica o la justicia social (Tusell, 2015). Autores (e.g., Diamond \& Morlino, 2004) plantean que la democracia ha dado un giro y debe atender temas como las políticas públicas, la igualdad política y el buen gobierno. De la misma forma, Escobar (2011) se suma a la idea de comprender la democracia como un ordenamiento estable que a través de las instituciones persiga la libertad e igualdad de sus ciudadanos, preocupándose de la responsabilidad política, el accountability y la legitimidad del sistema. Finalmente, otros (Barreda, 2011; Mainwaring \& Pérez-Liñán, 2008) plantean la relevancia que toma el crecimiento económico para los países y la sociedad, ya sea de forma positiva o negativa respecto a la calidad democrática.

La propuesta de Dahl (1989) conocida como poliarquía ha sido la más aceptada para explicar cómo debería ser la democracia y su funcionamiento perfecto. El autor propone tres grandes ejes de oportunidades referidos a (i) formular las preferencias; (ii) manifestar las preferencias; y (iii) recibir igualdad de trato por parte del gobierno en la ponderación de las preferencias. Cada uno de estos ejes requiere de ciertas garantías constitucionales para asegurar a cabalidad su calidad como la libertad de asociación, expresión, voto, libertad para que los líderes políticos compitan en busca de apoyo, diversidad de fuentes de información, elecciones libres e imparciales e instituciones que garanticen que la política de gobierno dependa de los votos y demás formas de expresar preferencias. Compartimos en cierta medida la visión de Dahl (1989) en cuanto a que la democracia debiese cumplir con todas las características mencionadas anteriormente, pero cuando la vemos en la práctica es evidente que hay distintos grados de desempeño que la alejan de su objetivo ideal. Por ende, la calidad de la democracia dependerá principalmente del cumplimiento de categorías mínimas o básicas que cercioren su buen funcionamiento, a pesar de que no asegura con totalidad una democracia plena. En consecuencia, entendemos que la democracia es un fenómeno complejo que se ha visto influenciado por diversos conflictos y hechos que le han impedido consolidarse plenamente como proyecto político.

Tusell (2015), plantea que los países del norte de Europa (e.g., Dinamarca, Finlandia, Suecia, Noruega y Suiza) tienen una mejor calidad de democracia que los Estados del sur, lo cual no resulta una coincidencia. La sociología ha escrito largamente sobre la democracia orientada a la conformación, transición y consolidación de la misma, problematizando acerca de la noción de modernización encauzada al progreso y desarrollo capitalista que representan los parámetros eurocéntricos. En una primera instancia se centró la mirada en ejes del tipo institucional, niveles macro de análisis -como factores estructurales- para luego girar en torno a estudios orientados en los procesos que implican el desarrollo y funcionamiento del sistema democrático, niveles 
Cereceda-Marambio, K., \& Torres-Solís, A. (2017). Satisfacción con la democracia en Chile: De lo normativo a lo valorativo. Revista de Sociología 32(1), 32-49. doi: 10.5354/0719-529x.2017.47884

micro centrados los cambios institucionales y las estrategias llevadas a cabo para su realización (Pérez-Liñán, 1998). La modernidad como proyecto ha sido el discurso dominante de los Estados del norte en vista de lo cual los Estados del sur deben ser su reflejo. Así lo plantea Quijano (2000), al poseer rasgos históricos europeos, aspectos materiales e intersubjetivos pero siendo a la vez profundamente distintos. Tal hecho muestra que, frente a la historia latinoamericana, las democracias del sur distan mucho de su ideal del norte.

Sin duda la tensión que subyace a la decisión tiene que ver con el inacabado debate sobre la democracia, su naturaleza cambiante versus la primacía de su esencia. La calidad de la democracia es una cuestión compleja que no goza de un consenso elemental respecto a su definición (Alcántara, 2008; Hagopian, 2005; Munck, 2004). Ciertamente, este término puede estar asociado a concepciones muy dispares de democracia: desde aquellas más minimalistas, que ponen el acento en los procedimientos básicos de una democracia a aquellas que incorporan mayores exigencias normativas (Barreda, 2011).

En este marco, es preciso observar que los regímenes democráticos se han consolidado en América Latina durante las últimas décadas luego de la caída de los regímenes autoritarios (Payne, Zovatto, \& Mateo, 2006). Sin embargo, la extensión de la democracia no significó estructuras gubernamentales más eficientes ni eficaces automáticamente, y la diferencia entre ellos hace que la atención se dirija hacia las características de las nuevas democracias (Barreda, 2011). Para Payne, Zovatto, y Mateo (2006) en las regiones en vías de desarrollo como Latinoamérica no solo adquieren importancia los factores económicos en el avance de la sociedad, si no que los factores políticos tienen un carácter central, específicamente el de la calidad de la política democrática. En este sentido, pese a la ola de democratización en América latina, ésta no cuenta con un apoyo elevado en la región (Hagopian, 2005).

Garretón (2004) señala que existen dos tipos de déficit en las democracias latinoamericanas: uno ligado a la transición a la democracia y otro a la insatisfacción con las democratizaciones. Lo anterior guarda relación con las herencias de los regímenes autoritarios y las negociaciones cupulares desembocando en la desvalorización del sistema político. En esto coincide con la idea de Morlino (2007) quien establece como un aspecto de importancia en la calidad de la democracia las ideas de las tradiciones autoritarias, es decir, los modelos introducidos o reforzados por el régimen autoritario anterior a la transición. Así, los regímenes democráticos latinoamericanos "en muchos casos están demasiado impregnadas de herencias institucionales y éticas de las dictaduras". (Garretón, 2004, p. 180). De la misma manera Avendaño (2011) se refiere a la incapacidad de las autoridades para subordinar a los militares al poder civil, por lo que la presencia de una tradición autoritaria ha restringido el normal funcionamiento de la democracia. La herencia no sólo es institucional, sino también se ha 

normativo a lo valorativo. Revista de Sociología 32(1), 32-49. doi: 10.5354/0719-529x.2017.47884

impregnado en el imaginario colectivo de la sociedad.

Se presentan diversos factores que crean grandes diferencias en la calidad de la democracia, comparando América Latina respecto al viejo continente, por lo cual es menester considerar explicaciones de distintas corrientes. En el caso chileno existen diferentes aspectos que afectan la calidad de la democracia, primero, el modelo neoliberal dejó de ser una forma de desarrollo, así, a pesar de los logros socio-económicos alcanzados por el país, no disminuye la brecha de la desigualdad; segundo, los actores del desarrollo no tiene como actor a la clase empresarial con responsabilidades frente al país, así como también se presenta una cierta timidez del Estado como actor; y tercero, el alejamiento producido entre el Estado y la política -como sistema de representación- de la sociedad civil (Garretón, 2004). Los intentos parciales e inconsistencias de la política para superar los aspectos heredados de la dictadura no han proveído de una base ética sólida para asegurar la consolidación de la democracia chilena.

Bajo la misma línea Castells (1999) plantea que el déficit democrático se ha visto asociado a los procesos de globalización producto que el Estado se ha convertido en un agente globalizador orientado a promover las pautas organizacionales que propone la modernidad y el progreso más que el ser el ente encargado de garantizar la democracia, representación, participación y debate interno ocasionando que pierda legitimidad frente a la sociedad civil. La democracia es más que asegurar la condición de elecciones libres, competitivas e imparciales sino más sustantivamente en acercar el Estado al ciudadano.

Finalmente, podemos decir que la legitimidad de la democracia y la calidad de esta tienen diversos factores que lo explican, tanto estructurales, políticos, sociales e históricos. En este debate la satisfacción con la democracia es uno de los aspectos que se liga a ella, por lo cual estudiarla en el contexto chileno actual permite poner a prueba el modelo teórico que más adelante se propone, explicar qué factores la explican, y cuáles no, además de analizar los rasgos que muestra sobre la población.

\section{¿Qué explica la satisfacción con la democracia?}

La satisfacción con la democracia ha sido uno de los conceptos más usados para el análisis político de esta, sin embargo, no existe consenso respecto a las dimensiones que este concepto involucra (Canache, Mondak, \& Seligson, 2001). Es por tal razón que existen diversas teorías dedicadas a tematizar la satisfacción con la democracia. Por ejemplo, Almond y Verba (1963) destacan la confianza social y la confianza en instituciones políticas y del Estado, y su relación con la legitimidad y satisfacción con el orden democrático. Así, cuando los ciudadanos expresan altos niveles de confianza en dichas instituciones existirá una mayor satisfacción con el sistema democrático en sí. Los regímenes democráticos se encuentran ligados al prestigio de las instituciones, dado que cada sociedad posee un orden particular constituido por diversas 

normativo a lo valorativo. Revista de Sociología 32(1), 32-49. doi: 10.5354/0719-529x.2017.47884

instituciones políticas, económicas, sociales y culturales que cumplen determinadas funciones, por lo que es importante la valoración que dan los ciudadanos a estas instituciones como parte misma del proceso de construcción de la democracia. Así, las instituciones sirven como medios de integración y participación de los sujetos al orden político (Huneeus, 1987).

Dada la relevancia que cumplen las instituciones dentro de los estados, es fundamental comprender la confianza o valoración que los ciudadanos hacen sobre las instituciones centrales del sistema político (Lipset \& Schneider, 1983). En términos de Levi y Stoker (2000) la confianza supone una evaluación positiva de los atributos más relevantes que vuelve a cada institución justa, creíble y transparente. Es decir, la confianza reside en aquel sentimiento primordial sobre el sistema político, el funcionamiento que posee y la capacidad de sentar bases sobre la igualdad entre los individuos al mismo tiempo de asegurarla. En esta línea, siguiendo a Huneeus (1987), entenderemos la confianza como el apoyo a una institución que implica hacer creíble sus actos y su discurso, pues las evaluaciones de las instituciones están estrechamente vinculadas a la satisfacción con la democracia.

Easton (1953) considera la satisfacción política en el sentido negativo del concepto, refiriéndose al descontento político el cual es consistente en los juicios de los ciudadanos sobre el comportamiento diario de los líderes políticos y el funcionamiento de los procesos e instituciones gubernamentales; lo cual coincide con la idea de que las la satisfacción con la democracia y las instituciones son inseparables (Karp \& Banducci, 2003). Easton (1953) distingue entre el apoyo difuso y apoyo específico a la democracia, siendo el primero una predisposición constituida por actitudes favorables que permiten a los ciudadanos aceptar o tolerar políticas contrarias a sus intereses, y el segundo la evaluación realizada sobre la base del desempeño de las instituciones y los líderes.

Consistentemente, Anderson y Guillory (1997) afirman la importancia del carácter institucional y económico, pero agregando la idea de la importancia de la economía y de las preferencias políticas de las personas. En este sentido, los autores plantean que la economía es vista como uno de los determinantes primarios hacia el proceso democrático encontrándose directa relación con el impacto en la satisfacción con la democracia por parte de los individuos. De este modo, la situación económica actual y futura son los factores fundamentales para explicar la valoración que dan los ciudadanos a la democracia. Siguiendo esta línea, Shumpeter (1942) y Sen (1999) son los principales precursores de la idea de que el orden democrático va ligado al crecimiento de la economía como símbolo de estabilidad de las instituciones y capacidad de avance conforme a los tiempos modernos. Así, esta tradición teórica ha propuesto largamente la importancia de la situación económica del país como el factor más influyente en la satisfacción con la democracia, mostrando cuánto funciona o no el sistema 
Cereceda-Marambio, K., \& Torres-Solís, A. (2017). Satisfacción con la democracia en Chile: De lo normativo a lo valorativo. Revista de Sociología 32(1), 32-49. doi: 10.5354/0719-529x.2017.47884

político. Por tal razón, si la ciudadanía percibe que la economía va en descenso, sentirá afectada su situación personal lo que se reflejará en críticas al modelo e insatisfacción con este. Siguiendo esta línea, hipotetizamos que cuando la percepción sobre el estado de la economía es desfavorable para el país (situación sociotrópica) o para las personas (situación egotrópica) habría una menor satisfacción con la democracia (Anderson \& Guillory, 1997; Hofferbert \& Klingemann, 1999).

Algunos autores (e.g., Anderson \& Guillory, 1997) hacen referencia a la importancia de las preferencias políticas de los ciudadanos y su influencia en la satisfacción con la democracia. Así, aquellas personas cuyo sector político (al que se sienten cercanos o por el que han votado) haya ganado las elecciones, se muestran más satisfechas con la democracia que aquellos denominados perdedores del proceso electoral (Díaz, 2014), lo cual revela la importancia de las preferencias políticas o la proximidad a un partido político o candidato/a en la satisfacción con la democracia. Paralelo a la relevancia de la percepción del funcionamiento de la economía, Hofferbert y Klingemann (1999) abordan la relevancia la percepción del grado en que las libertades y derechos políticos son respetados por el sistema, sindicando a esta última la más importante en sus estudios en Europa. En esta línea, algunos estudios (e.g., O’Donell, 2000) han mostrado la importancia de la relación entre la satisfacción con la democrática, y el bienestar económico, la institucionalidad y el bienestar con el individuo, incluyendo la percepción respeto por los derechos y libertades de la población, por lo cual, resulta importante considerar tales factores a la hora de analizar la satisfacción con la democracia más allá del ámbito institucional, orientando el análisis al sentir de los individuos respecto de la sociedad en que viven pues, al ver mermados sus derechos $\mathrm{y}$ perciben que el sistema falla $\mathrm{y}$ muestran su malestar con el sistema político y social.

Por otro lado, Sanhueza, Cea y Guerrero (2015) describen la importancia de integrar la noción de corrupción al análisis de la satisfacción con la democracia pues los sujetos que "tienen alta percepción de corrupción, [...] se muestran abiertamente insatisfechos con su funcionamiento, por lo que los niveles de corrupción no sólo dañan al gobierno de turno sino que también deprimen los índices de satisfacción con la democracia" (p. 93).

A esto, Morales (2009) propone que la percepción de la corrupción responde a una imagen que se crea respecto al desempeño de las autoridades, cuestión que adquiere especial relevancia en América Latina, pues el presidencialismo caracteriza las democracias de la región (Vairo, 2012). Tal hecho puede reflejar un malestar que manifiesta la falta de satisfacción con la democracia frente a la valoración del presidente en el país.

En síntesis, en la presente investigación proponemos que la satisfacción con la democracia no sólo se explica por el quehacer político y la estabilidad o crecimiento económico de un país, sino que también por aspectos que dicen relación con la percepción de los derechos políticos-civiles y los valores de la igualdad. De 
este modo, consideramos que es posible explicar la satisfacción con la democracia con un modelo basado en siete variables predictoras: (a) la confianza en las instituciones; (b) la percepción de la situación económica país; (c) la percepción de la situación económica personal; (d) preferencia política; (e) la percepción de la corrupción; (f) la percepción de la garantía de los derechos y libertades y; (g) la valoración presidencial.
Planteamos que estas siete variables tienen efectos directos sobre la satisfacción con la democracia y, a partir de ellas, es posible generar un modelo teórico-estadístico que denominaremos "modelo integrado de satisfacción con la democracia” que será contrastado empíricamente y permitirá comprender mejor la influencia de factores personales, valorativos y normativos sobre la satisfacción con la democracia en Chile (ver Figura 1).

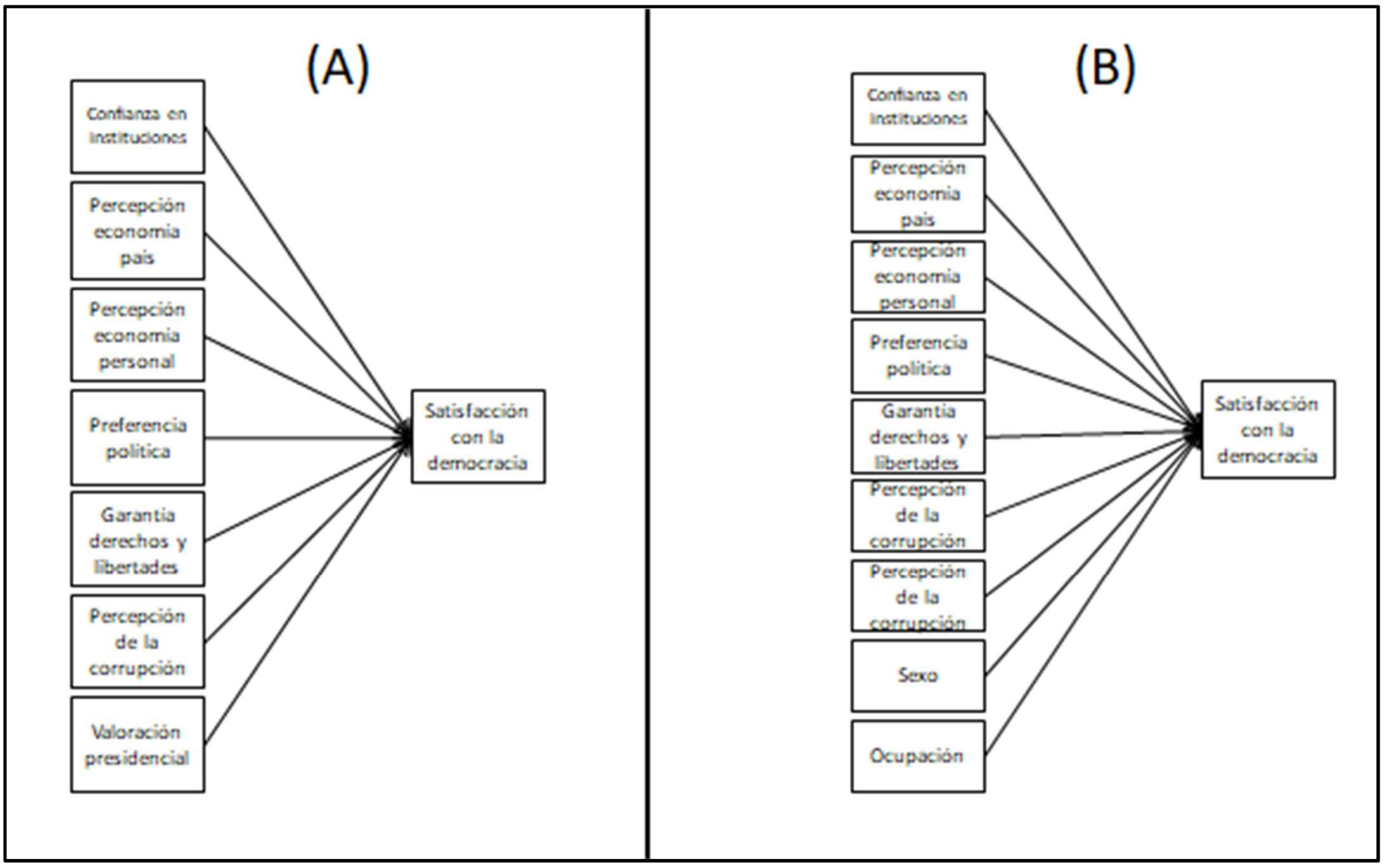

Figura 1. Modelo teórico (A) y modelo comparación (B). La flecha unidireccional muestra una relación causal desde la variable independiente o exógena hacia la dependiente o endógena. 


\section{MÉTODO}

Para contrastar el modelo propuesto se utilizó la base de datos de la encuesta Latinobarómetro 2015, la cual busca representar la satisfacción con la democracia de la población chilena en el año 2015, bajo el mandato de Bachelet. La muestra es de tipo probabilística en tres etapas, con una margen de error de \pm 2.8 . La muestra de la encuesta corresponde a 1,200 casos, en nuestro estudio luego de un proceso de recodificación de las categorías se trabajó con un total de 852 sujetos, hombres y mujeres mayores de 18 años, habitantes de Chile.

Para medir la variable dependiente y las independientes se construyeron una serie de índices a partir de las preguntas incluidas en la encuesta.

La variable satisfacción con la democracia fue medida a través de un índice construido a partir de tres indicadores que medían la percepción del grado democrático del país, satisfacción con el funcionamiento de la democracia y percepción de la democracia como el mejor sistema de gobierno. Todos los indicadores fueron transformados en escalas de uno a cuatro, y los indicadores negativos fueron invertidos de modo que puntajes más altos indican mayor satisfacción con la democracia. Posteriormente, los tres indicadores fueron sumados para construir la variable, la cual podía asumir valores entre 3 y 12 .

La variable confianza en las instituciones fue medida a través de un conjunto de preguntas relacionadas a cuánta confianza tienen los sujetos en: en las FFAA, policía/carabineros, el congreso, el gobierno, el poder judicial, los partidos políticos y el Estado. El fin era saber si la confianza en las instituciones formales influye en el grado de satisfacción con la democracia. Para esta variable se recodificaron todas las opciones cambiando el sentido de los valores, así puntajes más altos indican mayor confianza y menos puntaje, menor el nivel de confianza. Se asignó valores según cuánta confianza sintieran los sujetos con cada institución mencionada. Se utilizó la siguiente escala: mucha (4), algo (3), poca (2), o ninguna (1), para luego construir un índice con la suma de estos puntajes.

La variable percepción de la situación económica del país fue medida a través de un índice construido a partir de tres indicadores que guardaban relación con la satisfacción de la economía del país, la situación económica actual y la situación de la economía país en el futuro. Para garantizar la equivalencia entre las tres variables, dos de ellas fueron recodificadas en cuatro categorías, también se invirtieron los valores de modo que mayor puntaje indicara una mejor percepción de la situación económica del país. Luego, los tres indicadores fueron sumados para construir la variable de modo que el índice podía asumir valores entre tres y 12 . Se utilizó la siguiente escala que va desde muy buena (4) a muy mala (1).

La variable percepción de la situación económica personal fue medida a partir de tres preguntas orientadas que indicaban la situación económica actual del entrevistado y la familia, 
la situación económica a doce meses del entrevistado y su familia y si el salario del entrevistado más el ingreso total familiar les permite cubrir las necesidades. Para resguardar las equivalencias entre las variables se recodificaron dos de estas las cuales tenían cinco valores, para luego ser estandarizadas en cuatro categorías de respuesta. Finalmente se realizó una sumatoria de las tres variables para crear un índice el cual podía asumir valores entre tres y 12. Se utilizó la siguiente escala que va desde muy buena (4), a muy mala (1).

La variable percepción de la garantía de los derechos y libertades fue medida a partir de un índice conformado por 12 indicadores tales como libertad para participar en política, libertad para elegir mi oficio/profesión, protección del medio ambiente, protección de la propiedad privada, justa distribución de la riqueza, igualdad entre hombres y mujeres, igualdad de oportunidades sin importar el origen de cada cual, libertad de expresión siempre y en todas partes, libertad de profesar cualquier religión, protección contra el crimen, seguridad social, solidaridad con los pobres y los necesitados y oportunidades de conseguir trabajo. Cabe decir que todas las variables se recodificaron cambiando el sentido de sus valores, luego se sumaron y se creó un índice referente a las garantías de los derechos y las libertades el cual podía asumir valores entre 13 y 52 . Se utilizó la siguiente escala que va desde completamente garantizadas (4) a para nada garantizadas. Los estadísticos descriptivos de las variables cuantitativas del estudio se presentan en la Tabla 1.

Tabla 1. Estadísticos descriptivos de las variables cuantitativas del modelo

\begin{tabular}{|c|c|c|c|c|c|}
\hline & SCD & CIN & EPS & EPN & $\mathrm{DL}$ \\
\hline Media & 8.00 & 15.70 & 6.40 & 7.08 & 32.50 \\
\hline Desv. Estándar & 1.86 & 4.10 & 1.74 & 1.58 & 7.25 \\
\hline Mínimo & 3.00 & 7.00 & 3.00 & 3.00 & 13.00 \\
\hline Máximo & 12.00 & 28.00 & 12.00 & 12.00 & 52.00 \\
\hline Error típico (SE) & 0.05 & 0.12 & 0.05 & 0.04 & 0.22 \\
\hline Lim. Sup. & 8.28 & 15.90 & 6.51 & 7.17 & 32.90 \\
\hline Lim. Inf. & 8.05 & 15.40 & 6.30 & 6.99 & 32.00 \\
\hline
\end{tabular}

Nota: SCD: Satisfacción con la democracia. CIN = Confianza en las instituciones. EPS = Percepción de la Economía Personal. EPN = Percepción de la Economía País. DL = Garantía de Derechos y Libertades. IC 95\% = Intervalo de confianza al 95\%. Lim. Sup. = Límite superior. Lim Inf. = Límite inferior.

Para la variable preferencia política se utilizó la pregunta en la cual se pedía a los sujetos posicionarse en una escala de 0 a 10 , donde el valor cero indica posiciones políticas de izquierda y diez de derecha. Esta variable se convirtió en dummy, por ende se obtuvo 3 categorías: izquierda, derecha y otros. Para esto se agrupó la izquierda a los valores que iban de 0 a 5, la derecha a aquellos entre el 6 y 10 y otros a los no sabe/no responde. Sólo se encontró una pregunta referida al tema en la base de datos, presentándose una limitación 
Cereceda-Marambio, K., \& Torres-Solís, A. (2017). Satisfacción con la democracia en Chile: De lo normativo a lo valorativo. Revista de Sociología 32(1), 32-49. doi: 10.5354/0719-529x.2017.47884

metodológica por lo cual se asume el posible sesgo que esto implica. La dummy "izquierda" presentó una media de .46 puntos $(\mathrm{SD}=.49$; $\mathrm{SE}=.01$; IC 95\% [.43; .49]); la dummy "derecha" indicó una media de .16 puntos $(\mathrm{SD}=.36 ; \mathrm{SE}=$ .01 ; IC 95\% [.14;.18]); y por último la dummy de referencia mostró una media de .37 puntos $(\mathrm{SD}=.48: \mathrm{SE}=.01 ; \mathrm{IC} 95 \%[.34 ; .40])$.

En cuanto a la variable valoración presidencial se midió a partir de la pregunta referida a la aprobación o desaprobación de la gestión del presidente durante el año que se realizó la encuesta. Se transformó en variable dummy obteniendo 3 categorías: aprueba, desaprueba y otros a los no sabe/no responde. Sólo se encontró una pregunta referida al tema en la base de datos, presentándose una limitación metodológica por lo cual se asume el posible sesgo que esto implica. Primero, para la dummy "aprueba" presentó una media de .48 puntos $(\mathrm{SD}=.50 ; \mathrm{SE}=.01 ; \mathrm{IC} 95 \%[.45, .51]) ;$ para la dummy "desaprueba" indicó una media de .35 $(\mathrm{SD}=.47 ; \mathrm{SE}=.01 ; \mathrm{IC} 95 \%[.32, .37])$ y para la dummy "otros" mostró una media de .16 (SD = $.36 ; \mathrm{SE}=0.01 ; \mathrm{IC} 95 \%[0.14,0.18])$.

La variable percepción de la corrupción fue analizada a partir de la pregunta cuánto se cree que se había trabajado en reducir la corrupción de las instituciones del Estado en los últimos dos años. Se transformó en variable dummy obteniendo 3 categorías: mucho, poco y otros a los no sabe/no responde. Sólo se encontró una pregunta referida al tema en la base de datos, presentándose una limitación metodológica por lo cual se asume el posible sesgo que esto implica. Para la dummy "mucho" se presentó una media de 0.36 puntos $(\mathrm{SD}=0.48 ; \mathrm{SE}=0.01$; IC 95\% [0.34, 0.39]); para la dummy "poco" se indicó una media de 0.54 puntos $(\mathrm{SD}=0.49 ; \mathrm{SE}$ $=0.01$; IC 95\% [0.51, 0.57]) y para la dummy llamada "otros" indicó una media de 0.08 puntos $(\mathrm{SD}=0.28 ; \mathrm{SE}=0.008 ; \mathrm{IC} 95 \%[0.07,0.10])$.

Para las variables de control se decidió introducir el sexo del entrevistado como variable dummy, donde "mujer" presentó una media de 0.52 puntos $(\mathrm{SD}=0.49 ; \mathrm{SE}=0.01 ; \mathrm{IC}$ $95 \%$ [0.49, 0.55]). En cuanto a la segunda variable de control se decidió introducir la ocupación del entrevistado la cual poseía 7 categorías: independiente/cuenta propia (1); asalariado empresa pública (2); asalariado empresa privada (3); temporalmente no trabaja (4); retirado-pensionado (5); no trabajaresponsable de las compras y el cuidado de la casa (6); y estudiante (7). Esta variable presentó una media de 3.78 puntos $(\mathrm{SD}=1.85 ; \mathrm{SE}=0.05$; IC 95\% [3.36, 3.38])

La finalidad de incluir las variables de control reside en evaluar la robustez de las relaciones entre las variables independientes con la variable dependiente.

La relación entre satisfacción con la democracia y las dimensiones propuestas fue analizada a partir de una regresión lineal múltiple. Previo al análisis de regresión lineal múltiple, se evaluó mediante técnicas gráficas la condición de linealidad y con análisis de covarianza la condición de ausencia de colinealidad. Los resultados preliminares permitieron determinar que ambas condiciones de aplicación se cumplían. 


\section{RESULTADOS}

En conjunto, las variables independientes del modelo están altamente relacionadas con la satisfacción con la democracia $(\mathrm{R}=.641) \mathrm{y}$ tienen una varianza explicada alta $\left(\mathrm{R}^{2}\right.$ ajustado $=.404$ ) con una dispersión de los puntos en torno al hiperplano multidimensional de 1.48 puntos en promedio. En el caso del modelo de contraste tenemos resultados muy similares. $\mathrm{Al}$ incluir las variables de control, en conjunto con las 7 variables, muestran una relación levemente más alta $(\mathrm{R}=.649)$ con la satisfacción con la democracia que en el modelo integrado, con una varianza explicada $\left(\mathrm{R}^{2}\right.$ ajustado $=.412)$ y una dispersión en el hiperplano de regresión $(\mathrm{SE}=1.47)$ de similar magnitud al modelo integrado, por lo que podemos afirmar que el incorporar las 2 variables de control al modelo (en este caso: sexo y ocupación) no contribuye a mejorar sustancialmente la capacidad predictiva del modelo. Pese a lo anterior, tanto el modelo integrado como el modelo de contraste mostraron una distribución no normal de los residuales (Modelo integrado: K-S $=.038, d f=$ 852, $p=.006$; Modelo de contraste: K-S $=.040$, $d f=852, \mathrm{p}=.002)$, lo cual estaría mostrando la potencial ausencia de algunas variables relevantes en el modelo.

Los resultados de los parámetros estimados en el modelo integrado y el de contraste se muestran en la Tabla 2. En ella, se observa un efecto positivo de la confianza en las instituciones en la satisfacción con la democracia $(b=0.137, p<.001)$ de intensidad moderada $(B=.283)$; resultados que no variaron sustancialmente al incorporar las variables de control (ver modelo de contraste). Esto significa que a medida que aumente la confianza en las instituciones mayor será la satisfacción de la democracia; resultados que concuerdan con la hipótesis del estudio. Una situación similar ocurre con la percepción de la economía país que mostró un efecto positivo $(b=0.405, \mathrm{p}<.001) \mathrm{de}$ intensidad moderada $(B=.368)$ sobre la satisfacción con la democracia, resultados que se mantuvieron en el modelo de contraste, lo que indica que a medida que mejora la percepción de la economía del país la satisfacción de la democracia tiende a mejorar. Por su parte, la percepción de la economía personal no mostró un efecto significativo en la satisfacción con la democracia $(b=0.005, p>$ $.05, B=.004)$, lo que refuta la hipótesis de este estudio Este resultado contrasta con el resultado observado para la percepción de la situación económica país, lo que podría deberse, probablemente, a que la situación económica personal no es entendida por los sujetos como un fenómeno vinculado al sistema político democrático, sino como un fenómeno meramente individual, ligado a la idea que el individuo es el responsable por su propia movilidad social (Arteaga \& Pérez, 2011; Boccardo \& Ruiz, 2015), desligando los aspectos más estructurales, como el sistema económico imperante o sistema político regente -régimen democrático- de la situación económica personal. 
Tabla 2. Coeficientes estimados de los modelos predictivos de la Satisfacción Democrática.

\begin{tabular}{|c|c|c|c|c|c|c|c|c|}
\hline & \multicolumn{4}{|c|}{ Modelo Teórico } & \multicolumn{4}{|c|}{ Modelo de Contraste } \\
\hline & \multirow[t]{2}{*}{$\mathrm{B}(\mathrm{SE})$} & \multicolumn{2}{|c|}{ I.C.(B) $95 \%$} & \multirow[t]{2}{*}{$\operatorname{Std}(B)$} & \multirow[t]{2}{*}{$\mathrm{B}(\mathrm{SE})$} & \multicolumn{2}{|c|}{ I.C.(B) $95 \%$} & \multirow[t]{2}{*}{$\operatorname{Std}(B)$} \\
\hline & & L. Inf & L. Sup & & & L.Inf. & L. Sup & \\
\hline Constante & $\begin{array}{l}3.281^{* *} \\
(.431)\end{array}$ & 2.434 & 4.128 & & $\begin{array}{l}2.743^{* *} \\
(.457)\end{array}$ & 1.846 & 3.640 & \\
\hline Confianza en las instituciones & $\begin{array}{l}.137 * * \\
(.016)\end{array}$ & .105 & .196 & .283 & $\begin{array}{l}.133^{* *} \\
(.016)\end{array}$ & 1.01 & .166 & .275 \\
\hline Percepción economía país & $\begin{array}{l}.405^{* *} \\
(.041)\end{array}$ & .325 & .485 & .368 & $\begin{array}{l}.401^{* *} \\
(.041)\end{array}$ & .320 & .481 & .365 \\
\hline Percepción economía personal & $\begin{array}{l}.005 \\
(.040)\end{array}$ & -.074 & .084 & .004 & $\begin{array}{l}.009 \\
(.040)\end{array}$ & .070 & .088 & .008 \\
\hline $\begin{array}{l}\text { Garantía de los derechos y } \\
\text { libertades }\end{array}$ & $\begin{array}{l}.026 * * \\
(.007)\end{array}$ & .011 & .040 & .099 & $\begin{array}{l}.028^{* *} \\
(.007)\end{array}$ & .013 & .042 & .106 \\
\hline Preferencia política & & & & & & & & \\
\hline D1: Izquierda & $\begin{array}{l}.296 * \\
(.130)\end{array}$ & .040 & .552 & .077 & $\begin{array}{l}.316^{*} \\
(.130)\end{array}$ & .062 & .571 & .082 \\
\hline D2: Derecha & $\begin{array}{l}-.156 \\
(.162)\end{array}$ & -.474 & .162 & -.030 & $\begin{array}{l}-.083 \\
(.162)\end{array}$ & -.402 & .235 & -.016 \\
\hline \multicolumn{9}{|l|}{ Percepción corrupción } \\
\hline D1: Mucho disminuir & $\begin{array}{l}-.346 \\
(.268)\end{array}$ & -.873 & .181 & -.088 & $\begin{array}{l}-.262 \\
(.268)\end{array}$ & -.787 & .263 & -.067 \\
\hline D2: Poco por disminuir & $\begin{array}{l}-.294 \\
(.264)\end{array}$ & -.812 & .225 & -.076 & $\begin{array}{l}-.205 \\
(.264)\end{array}$ & -.723 & .313 & -.053 \\
\hline \multicolumn{9}{|l|}{ Valoración Presidente } \\
\hline D1: Aprueba & $\begin{array}{l}-.787^{* *} \\
(.173)\end{array}$ & -1.126 & -.447 & -.204 & $\begin{array}{l}-.730 * * \\
(.173)\end{array}$ & -1.068 & -.391 & -.189 \\
\hline D2: Desaprueba & $\begin{array}{l}-.836^{* *} \\
(.174)\end{array}$ & -1.117 & -.495 & -.207 & $\begin{array}{l}-.744^{* *} \\
(.175)\end{array}$ & -1.088 & -.400 & -.184 \\
\hline Sexo $(1=$ mujer $)$ & & & & & $\begin{array}{l}.220^{*} \\
(.111)\end{array}$ & .002 & .437 & .057 \\
\hline Ocupación & & & & & $\begin{array}{l}.071^{*} \\
(.030)\end{array}$ & .013 & .129 & .068 \\
\hline
\end{tabular}

Nota: $\mathrm{B}=$ Coeficiente de regresión. SE = Error típico. I.C. (B) 95\% = Intervalo de confianza al 95\% para el coeficiente de regresión. L. Inf. = Límite inferior del intervalo de confianza. L. Sup. = Límite superior del intervalo de confianza. Std (B):Coeficiente B de regresión estandarizado (Beta). ${ }^{* *}$ Coeficiente significativo al 99\% de confianza. * Coeficiente significativo al 95\% de confianza. Errores típicos se muestran entre paréntesis. 
Cereceda-Marambio, K., \& Torres-Solís, A. (2017). Satisfacción con la democracia en Chile: De lo normativo a lo valorativo. Revista de Sociología 32(1), 32-49. doi: 10.5354/0719-529x.2017.47884

La percepción de la garantía de los derechos y las libertades mostró un efecto positivo $(b=$ $0.026, \mathrm{p}<.001)$ aunque de baja intensidad $(B=$ .099) sobre la satisfacción con la democracia. En esto cabe mencionar que, a pesar de que se esperaba encontrar efectos relevantes de los aspectos valóricos relacionados al bienestar subjetivo en la satisfacción con la democracia, llama la atención que esta variable presente una relación relativamente baja con la dependiente, lo cual podría deberse a que cambio desde un régimen dictatorial a uno democrático producido relativamente recientemente, no ha logrado consolidar la relación y sociabilización de los valores democráticos, generando una democracia incompleta o imperfecta (Garretón, 2000). De manera similar, la identificación política con la izquierda tiene efectos positivos $(b=0.226, p<$ $.005)$ de baja intensidad $(B=.077)$ en la satisfacción con la democracia, comparado con las personas que se identificaban con la derecha y quienes no se identifican con ningún punto del continuo izquierda-derecha. En tanto que la percepción de la corrupción no mostró efectos significativos en la satisfacción con la democracia ( $b=-0.010, p>.05, B=-.001)$, lo que muestra evidencia contraria a lo planteado por teorías recientes sobre el tema (Sanhueza et al., 2015).
Finalmente, en cuanto a la valoración del presidente podemos observar que si bien nuestra hipótesis teórica era que una apoyo a la figura presidencial generaba mayor satisfacción con la democracia, en los resultados, se observó que quienes aprueban la gestión presidencial (b $=-0.787, \mathrm{p}<.001 ; \quad B=.204) \mathrm{y}$ quienes desaprueban la gestión presidencial $(b=-.836, p$ $<.001 ; B=.207)$ tienen niveles más bajos de satisfacción con la democracia que quienes no aprueban ni desaprueban la gestión presidencial. En otras palabras, los que no tienen una idea clara sobre la gestión presidencial son quienes tienen los niveles más altos de satisfacción con la democracia, pero estar de acuerdo con la gestión actual o estar en desacuerdo, disminuye estos niveles de satisfacción. Una posible explicación a este fenómeno podría ser que quienes desaprueban el gobierno se sienten menos satisfechos con una figura presidencial no es de su preferencia y quienes aprueban la gestión del gobierno pueden tener expectativas más altas sobre el funcionamiento de la democracia, lo que redunda en insatisfacción, en tanto que quienes no tienen una posición definida podrían restarle relevancia a la figura presidencial por lo cual su satisfacción con la democracia pasa por otros factores, evitando generar ilusiones frente a la labor de la máxima autoridad. 


\section{CONCLUSIONES}

La principal conclusión de la presente investigación fue constatar que el modelo integrado logra una adecuada capacidad explicativa de la satisfacción con la democracia, con una aproximación bastante parsimoniosa. Las variables que mostraron resultados significativos y relevantes (i.e., valoración del presidente, preferencia política, percepción de la económica del país, percepción de la garantía a derechos y libertades y la confianza en las instituciones) mantuvieron sus tamaños de efecto (ver coeficientes estandarizados en tabla 2) al introducir las variables de control, lo que muestra un importante grado de robustez de las relaciones observadas. Por lo tanto, comparativamente, modelo integrado es mejor que el modelo de contraste ya que las variables de control no generaron fluctuaciones en los parámetros estimados por el modelo y no contribuyeron sustancialmente a mejorar la predicción de la satisfacción con la democracia en la muestra analizada.

Las variables más importantes (i.e., con tamaños de efecto mayores) para explicar la satisfacción con la democracia son la percepción de la económica del país y la confianza en las instituciones; lo cual respalda los postulados de las teorías clásicas (Easton, 1953) sobre la satisfacción con la democracia.

En contraste, el presente estudio mostró algunos resultados no esperados respecto al efecto que tiene la aprobación del presidente. En esta variable, se observó que aquellos que indicaron no tenían una valoración positiva ni negativa del presidente fueron quienes mostraron tener mayores niveles de satisfacción con la democracia comparativamente. Lo anterior se puede deber a que los que desaprueban tienden a ser más críticos afectando su satisfacción personal con la democracia, mientras los que aprueban, consideramos, pueden tener una mayor información sobre la política como también formarse mayores ilusiones, siendo más críticos con las condiciones democráticas del país, repercutiendo su satisfacción con esta. En cambio, aquellos que no toman posición respecto a esta pregunta podrían estar menos informados políticamente, o simplemente no se crean expectativas respecto al funcionamiento de la democracia. Cabe destacar que esta situación sólo podemos aplicarla al caso chileno, por ende, sería interesante observar tal escenario a lo largo de Latinoamérica mediante la realización de un análisis comparado, dada la importancia de los mandatarios en el continente y la preeminencia del presidencialismo. Consideramos importante indagar en este tema más a fondo en futuras investigaciones, donde se tenga información suficiente que permita elaborar en detalle esta relación.

Finalmente, un hallazgo interesante de este estudio es la idea de la baja relación entre la percepción de la garantía derechos y libertades y la satisfacción con la democracia. Este resultado contrasta con lo mostrado en estudios en el continente europeo (cf. Hofferbert \& Klingemann, 1999), donde esta variable ha 
Cereceda-Marambio, K., \& Torres-Solís, A. (2017). Satisfacción con la democracia en Chile: De lo normativo a lo valorativo. Revista de Sociología 32(1), 32-49. doi: 10.5354/0719-529x.2017.47884

mostrado efectos significativos y de mayor magnitud a los observados en este estudio. Probablemente, estas diferencias se deban a las características particulares de la democracia latinoamericana y especialmente, la democracia chilena (Garretón, 2004); no obstante, debido a que la garantía de derechos y libertades constituye la base institucional ética y moral sobre la cual se sustentan los sistemas democráticos, es importante considerar este hallazgo como un signo de advertencia de una probable falta de consolidación de la democracia a nivel local y nacional, aspecto en el cual es necesario continuar indagando.

El presente estudio ha contribuido a delimitar un conjunto de variables que permite predecir la satisfacción con la democracia y se espera que estos hallazgos sean de utilidad para futuras investigaciones que busquen mejorar el modelo que hemos propuesto, por ejemplo, incorporando al modelo algún indicador de la percepción del accountability, es decir, la percepción de los mecanismos en que el gobierno y líderes políticos deben rendir cuentas a la sociedad civil (Barreda, 2011). Dicho aspecto es importante dado que trabajos recientes lo vinculan a la satisfacción con la democracia (cf. Aarts \& Thomasen, 2008), con lo cual consideramos que sería fructífero abordar dicho aspecto como una potencial mejora del modelo integral propuesto. En esta misma línea, consideramos que futuras investigaciones debiesen profundizar en la relación entre la corrupción y la satisfacción con la democracia y ahondar, mediante estudios longitudinales, en los efectos de las fluctuaciones económicas sobre la satisfacción con la democracia y/o profundizar en las diferencias y similitudes en la satisfacción con la democracia en contextos latinoamericanos y europeos, tomando en consideración la importancia de las teorías del desarrollo y las inflexiones decoloniales.

\section{REFERENCIAS}

Aarts, K., \& Thomassen, J. (2008). Satisfaction with democracy: Do institutions matter? Electoral Studies, 27, 5-18.

Alcántara, M. (2008). Luces y sombras de la calidad de la democracia en América Latina. Quórum. Revista de Pensamiento Iberoamericano, 22, 169-181.

Almond G., \& Verba S. (1963). The civic culture: Political attitudes and democracy in five nations. Princeton: Princeton University press.

Altman, D, \& Pérez-Liñán, A. (2002). Assessing the quality of democracy: freedom, competitiveness and participation in eighteen Latin American countries. Democratization, $9(2), 85-100$.

Anderson, J., \& Guillory, C. (1997). Political institutions and satisfaction with democracy: a cross-national analysis of consensusand majoritarian systems. American Political Science Review, 91, 66-81.

Arteaga, C. \& Pérez, S. (2011). Experiencias de vulnerabilidad: de las estrategias a las tácticas subjetivas. Universum (Talca), 26(2), 67-81. 
Cereceda-Marambio, K., \& Torres-Solís, A. (2017). Satisfacción con la democracia en Chile: De lo normativo a lo valorativo. Revista de Sociología 32(1), 32-49. doi: 10.5354/0719-529x.2017.47884

Avendaño, O. (2011). Calidad de la democracia y desempeño institucional en Chile. Un análisis retrospectivo en base a las propuestas del gobierno de Sebastián Piñera. Revista Política, 49(2), 127-149.

Barreda, M. (2011). La calidad de la democracia: Un análisis comparado de América Latina. Política y gobierno, 18(2), 265-295.

Boccardo, G., \& Ruiz, C. (2015) Los chilenos bajo el neoliberalismo. Clases y conflicto social. De Raíz Diversa, 5(3), 211-220.

Canache, D., Mondak, J. J., \& Seligson, M. A. (2001). Meaning and measurement in crossnational research on satisfaction with democracy. Public Opinion Quarterly, 65(4), 506-528.

Castells, M. (1999). Globalización, identidad y estado en América Latina. Santiago de Chile. PNUD.

Dahl, R. (1989). La poliarquía. Madrid: Tecnos.

Diamond, L. J., \& Morlino, L. (2004). The quality of democracy: an overview. Journal of democracy, 15(4), 20-31.

Díaz, A. M. (2014). La (in)satisfacción con el funcionamiento de la democracia en América Latina. Iberoamericana, 54, 169-173.

Easton, D. (1953) The political system. An inquiry into the state of political science. New York: Knopf Incorporated.

Escobar, M. (2011). La calidad democrática. Una propuesta para su medición por expertos. Reis: Revista Española de Investigaciones Sociológicas, 133, 59-80.

Garretón, M. (2004). De la transición a los problemas de calidad en la democracia chilena. Política, 42, 179-206.

Hagopian, F. (2005). Derechos, representación y la creciente calidad de la democracia en Brasil y Chile, Política y Gobierno, 12, 41-90.

Hofferbert, R., \& Klingemann, H. (1999) Remembering the bad old days: human rights, economic conditions, and democratic performance in transitional regimes.
European Journal of Political Research 36, 155-174.

Huneeus, C. (1987). Los chilenos y la política: cambio y continuidad bajo el autoritarismo. Santiago: CERC.

Huntington, S. P. (1994). La tercera ola: la democratización a finales del siglo $X X$. Barcelona: Paidos, Ibérica.

Karp, J., \& Banducci, S. (2003). To know it is love it? Satisfaction with democracy in the European Union. Comparative Political Studies. 36(3), 271-292.

Corporación Latinobarómetro (2015). Informe Latinobarómetro. Santiago de Chile.

Levi, M., \& Stoker, L. (2000). Political trust and trustworthiness. Annual Review of Political Science. 3, 475-507

Lipset, S., \& Schneider, W. (1983). The confidence gap: Business, labor, and government in the public mind. Nueva York: Free Press

Mainwaring, S., \& Pérez-Liñán, A. (2008). Regime legacies and democratization. explaining variance in the level of democracy in Latin America, 1978-2004. Kellogg Institute for International Studies.

Morales, M. (2009). Corrupción y democracia: América Latina en perspectiva comparada. Gestión y política pública, 18(2), 205-252.

Morlino, L. (2007). Explicar la calidad democrática: ¿qué tan relevantes son las tradiciones autoritarias?. Revista de Ciencia Política, 27, 3-22.

Munck, G. (2004). La política democrática en América Latina: contribuciones de una perspectiva institucional. Política y Gobierno, 25(2), 315-346.

O'Donnell, G. (2000). Teoría democrática y política comparada. Desarrollo Económico, 39(156), 519-570

Payne, J., Zovatto, M., \& Mateo, M. (2006). La Política Importa. Democracia y desarrollo en América Latina. Washington D.C.: Planeta. 
Pérez-Liñán, A. (1998). El estudio de la democracia en perspectiva comparada: Nuevas preguntas, viejas respuestas. Postdata, 3, 221-241.

Pierson, C. (2011). The modern state. New York: Routledge.

Quijano, A. (2000). Colonialidad del poder, eurocentrismo y América Latina. En E. Lander (Comp.), La colonialidad del saber: Eurocentrismo y ciencias sociales (pp. 201246). Buenos Aires: CLACSO.

Sanhueza, C., Cea, C., \& Guerrero, A. (2015). Democracia en Latinoamérica, ¿Qué factores influyen en la satisfacción y apoyo a la Democracia?. Revista de Ciencias Sociales Fronteras, 2, 85-113.

Schumpeter, J. (1942). Capitalismo, socialismo y democracia. Ciudad de México: Ed. Folio.
Sen, A. (1999). Desarrollo y libertad. Buenos Aires: Planeta.

Tusell, A. (2015). La calidad de la democracia y sus factores determinantes Un análisis comparado de 60 países. Política y Sociedad, 52, 179-205.

Vairo, D. (2012). "El consenso de los perdedores" y la legitimidad de la democracia en América del Sur. Política y gobierno, 19, 4169.

Manuscrito recibido: 08-08-2017

Corrección recibida: 19-09-2017

Manuscrito aceptado: 03-10-2017 